This document is the unedited Author's version of a Submitted Work that was subsequently accepted for publication in the

JOURNAL OF THE AMERICAN CHEMICAL SOCIETY, copyright (C) American Chemical Society after peer review.

To access the final edited and published work see https://pubs.acs.org/articlesonrequest/AOR-DnlhHw8Kdfn8HzpvqXdI

\title{
Supramolecular-enhanced charge-transfer within entangled polyamide chains as origin of the universal blue fluorescence of polymer carbon dots
}

Lorenzo Vallan, ${ }^{\dagger}$ Esteban P. Urriolabeitia, ${ }^{*}$ Fernando Ruipérez, ${ }^{\S}$ Jon M. Matxain, ${ }^{\#}$ Ruben Canton-Vitoria, ${ }^{\ddagger}$ Nikos Tagmatarchis, ${ }^{\ddagger}$ Ana M. Benito, ${ }^{\dagger}$ Wolfgang K. Maser ${ }^{* \dagger}$

${ }^{\dagger}$ Instituto de Carboquímica (ICB-CSIC), C/Miguel Luesma Castán 4, E-50018 Zaragoza, Spain.

*Instituto de Síntesis Química y Catálisis Homogénea (ISQCH-CSIC Universidad de Zaragoza), C/Pedro Cerbuna 12, E-50009 Zaragoza, Spain.

${ }^{\S}$ POLYMAT, University of the Basque Country UPV/EHU, Joxe Mari Korta Center, Avda. Tolosa 72, 20018 Donostia - San Sebastián, Spain.

\#Kimika Fakultatea, Euskal Herriko Unibertsitatea UPV/EHU and Donostia International Physics Center (DIPC), P.K. 1072, 20080, Donostia, Euskadi (Spain)

${ }^{¥}$ Theoretical and Physical Chemistry Institute, National Hellenic Research Foundation, 48 Vassileos

Constantinou Avenue, 11635 Athens, Greece 
Postprint: Unedited Author's version of Submitted Work published on-line on 13 Sept. 2018 in Journal of the American Chemical Society, DOI: 10.1021/jacs.8b06051

\title{
Supramolecular-enhanced charge-transfer within entangled polyamide chains as origin of the universal blue fluorescence of polymer carbon dots
}

\author{
Lorenzo Vallan, ${ }^{\dagger}$ Esteban P. Urriolabeitia, ${ }^{\ddagger}$ Fernando Ruipérez, ${ }^{\S}$ Jon M. Matxain, ${ }^{\#}$ Ruben Canton- \\ Vitoria, ${ }^{\ddagger}$ Nikos Tagmatarchis, ${ }^{\ddagger}$ Ana M. Benito, ${ }^{\dagger}$ Wolfgang K. Maser ${ }^{* \dagger}$ \\ †Instituto de Carboquímica (ICB-CSIC), C/Miguel Luesma Castán 4, E-50018 Zaragoza, Spain. \\ ${ }^{\ddagger}$ Instituto de Síntesis Química y Catálisis Homogénea (ISQCH-CSIC Universidad de Zaragoza), C/Pedro Cerbuna 12, E- \\ 50009 Zaragoza, Spain. \\ ${ }^{\S}$ POLYMAT, University of the Basque Country UPV/EHU, Joxe Mari Korta Center, Avda. Tolosa 72, 20018 Donostia - San \\ Sebastián, Spain. \\ "Kimika Fakultatea, Euskal Herriko Unibertsitatea UPV/EHU and Donostia International Physics Center (DIPC), P.K. 1072, \\ 20080, Donostia, Euskadi (Spain) \\ ${ }^{¥}$ Theoretical and Physical Chemistry Institute, National Hellenic Research Foundation, 48 Vassileos Constantinou Avenue, \\ 11635 Athens, Greece.
}

\begin{abstract}
The emission of a bright blue fluorescence is a unique feature common to the vast variety of polymer carbon dots (CDs) prepared from carboxylic acid and amine precursors. However, the difficulty to assign a precise chemical structure to this class of CDs yet hampers the comprehension of their underlying luminescence principle. In this work, we show that highly blue fluorescent model types of CDs can be prepared from citric acid and ethylenediamine through low temperature synthesis routes. Facilitating controlled polycondensation processes, the CDs reveal sizes of $1-1.5 \mathrm{~nm}$ formed by a compact network of short polyamide chains of about ten monomer units. Density functional theory calculations of these model CDs uncover the existence of spatially separated highest occupied molecular orbital and lowest unoccupied molecular orbital located at the amide and carboxylic groups, respectively. Photoinduced charge-transfer between these groups thus constitutes the origin of the strong blue fluorescence emission. Hydrogen-bond mediated supramolecular interactions between the polyamide chains enabling a rigid network structure further contribute to the enhancement of the radiative process. Moreover, the photoinduced charge-transfer processes in the polyamide network structure easily explain the performance of CDs in applications as revealed in studies on metal ion sensing. These findings thus are of general importance to the further development of polymer CDs with tailored properties as well as for the design of technological applications.
\end{abstract}

\section{INTRODUCTION}

Fluorescent carbon dots (CDs) are a unique class of spherical nano-meter sized objects contributing to the most recent advances in the fields of sensors, ${ }^{1-6}$ biosensors, ${ }^{7-10}$ bioimaging, ${ }^{11-14}$ drug delivery, ${ }^{15-18}$ light emitting devices, ${ }^{19-20}$ and photocatalysis. ${ }^{21-22}$ Apart of their high fluorescent quantum yields, their use in diverse applications is based on their facile fabrication and a broad set of cheap precursor materials, their water solubility, low toxicity and good biocompatibility, as well as their favorable energy and charge transfer properties. Unfortunately, the polydispersity of the $\mathrm{CD}$ products and the difficulty to ascertain a precise chemical structure yet undermine the comprehension of the underlying general fluorescence phenomena and also hinder the design of advanced CDs with tunable optical properties.

The synthesis of fluorescent CDs by common bottom-up approaches involves the polymerization/cross-linking of organic molecules and/or polymers at high temperature in hydrothermal or microwave-assisted reactions. ${ }^{23-25}$ The formation process resembles a dehydration and condensation process among the abundant functional groups of the precursor materials, whereby the resulting polymer CDs are composed of mixtures of molecules, oligomers, polymer chains, polymer clusters, which even may show a certain degree of carbonization, depending on the starting reagents and the synthesis conditions. ${ }^{26-27}$ Random reaction processes thus contribute to the vast number of $\mathrm{CD}$ products available. However, despite their large variety and uncertain chemical structure, all these types of CDs reveal a unique blue fluorescence emission as common unifying feature.

Part of recent studies relate the fluorescence of CDs to the detection of conjugated fluorophores, such as IPCA $(1,2,3,5-$ tetrahydro-5-oxo-imidazo[1,2- $\alpha$ ]pyridine-7-carboxylic acid), 28-30 derivatives or citrazinic acid ${ }^{31-32}$ or pyridine-like structures. $^{33-34}$ On the other hand, the group of B. Yang put under closer scrutiny the polymeric structure of the CDs. The observation that also non-conjugated polymers cause a bright photoluminescence, regardless of the presence of other emissive sources such as molecular fluorophores or a carbon core was ascribed to the so-called cross-link enhanced 
emission (CEE) effect. ${ }^{24,35-39}$ Here the rigidity of the polymer network, achieved by cross-linking, aggregation or immobilization decreases the vibrational and rotational freedom of subfluorophores such as $\mathrm{C}=\mathrm{O}, \mathrm{N}=\mathrm{O}, \mathrm{C}=\mathrm{N}$ heteroatom-containing double bonds, facilitating their radiative relaxation. Nevertheless, the exact nature of these subfluorophores and their role in the optical properties of nonconjugated polymer CDs is not well understood yet, mainly due to the lack of studies focusing on the synthesis of CDs to which a precise chemical structure can be assigned.

The aim of this work is to unambiguously identify the origin of the blue fluorescence, commonly observed in nonconjugated polymer CDs. To this end, model types of blue fluorescent CDs with a well-defined structure, consisting of commonly employed carboxylic acid and amine functional groups are synthesized. Citric acid and ethylenediamine are chosen as rather simple precursor molecules and three synthesis routes are designed to establish a polycondensation process resulting in amide bond formation while avoiding undesired side-reactions. This includes low temperature microwave assisted reactions controlling reaction time and reactive groups, as well as a novel yet unexplored roomtemperature synthesis route, exploiting reactions based on carbodiimide chemistry. All three routes produce highly blue emitting fluorescent polymer CDs of 1 to $1.5 \mathrm{~nm}$. A complete characterization of their chemical structure by a broad set of methods reveals the formation of a compact network of short polyamide chains of about ten monomer units. Density functional theory (DFT) analysis of the ground and excited state of these model polymer CDs disclose the existence of spatially separated highest occupied (HOMO) and lowest unoccupied (LUMO) molecular orbitals located at the amide and carboxylic moieties, respectively. Photoinduced chargetransfer between these groups thus constitutes the origin of the strong luminescence behavior, further assisted by hydrogenbond mediated supramolecular interactions of the entangled polyamide chains. Finally, studies on the use of polymer CDs for metal ion sensing further confirm that their performance is a direct consequence of the supramolecular polyamide network structure and the photoinduced charge-transfer processes between spatially separated amide and carboxylic groups.

\section{RESULTS AND DISCUSSION}

Citric acid (CA) and ethylenediamine (EDA) in a ratio 1:1 were chosen as starting material for the synthesis of CDs1. The fluorescent nanoparticles were obtained by microwave irradiation at $140^{\circ} \mathrm{C}$ for 3 minutes (see experimental part). A preliminary study shows that an increase of the reaction time in the considered range does not produce any change on the size, structure and optical properties of the product (see Supporting Information for details, Figures S1-S10 and Tables S1-S3). During the synthesis of CDs1 the amide bond formation is expected. The amidic condensation between $\mathrm{CA}$ and EDA thus could be considered as a sufficient condition for obtaining fluorescent CDs. In order to investigate the role of the amide bond in the formation of the fluorophore, two more syntheses of CDs were carried out. Keeping in mind that the $\mathrm{CA}$ reactivity is not only determined by the acid moieties, but also by the presence of the hydroxyl group, which at high temperature could lead to unpredictable products, CA was replaced by tricarballylic acid (TA). While this leaves unaffected the plausible formation of the amide bonds, it ensures that reactions involving the hydroxyl group are excluded. Thus, in a process equivalent to the synthesis of CDs1, the nanoparticles CDs2 were obtained from a water solution of TA and EDA 1:1 under microwave irradiation (see experimental part). The reaction temperature is an additional decisive parameter to keep under control the synthesis, since high temperatures enable a wider range of reaction pathways and reorganization processes. Therefore, a third material was prepared at room temperature, exploiting carbodiimide chemistry. Here the coupling agent $N, N^{\prime}$ diisopropylcarbodiimide was employed to selectively catalyze the condensation of CA and EDA (1:1 molar ratio) at room temperature (see experimental part) affording as product CDs3. Analyses of the three CDs samples by atomic force microscopy (Supporting Information, Figure S11), dynamic light scattering (Supporting Information, Figure S12 and Tables S4) and diffusion ordered spectroscopy (Supporting Information, Figure S13) reveal in all the cases the formation of nanoparticles with sizes between $1-1.6 \mathrm{~nm}$. Interestingly, all CDs samples show a bright blue emission when irradiated by UV light (Figure 1).

The optical properties of CDs1, CDs2 and CDs3 were studied by UV/Vis spectroscopy (Figure 2a), steady state

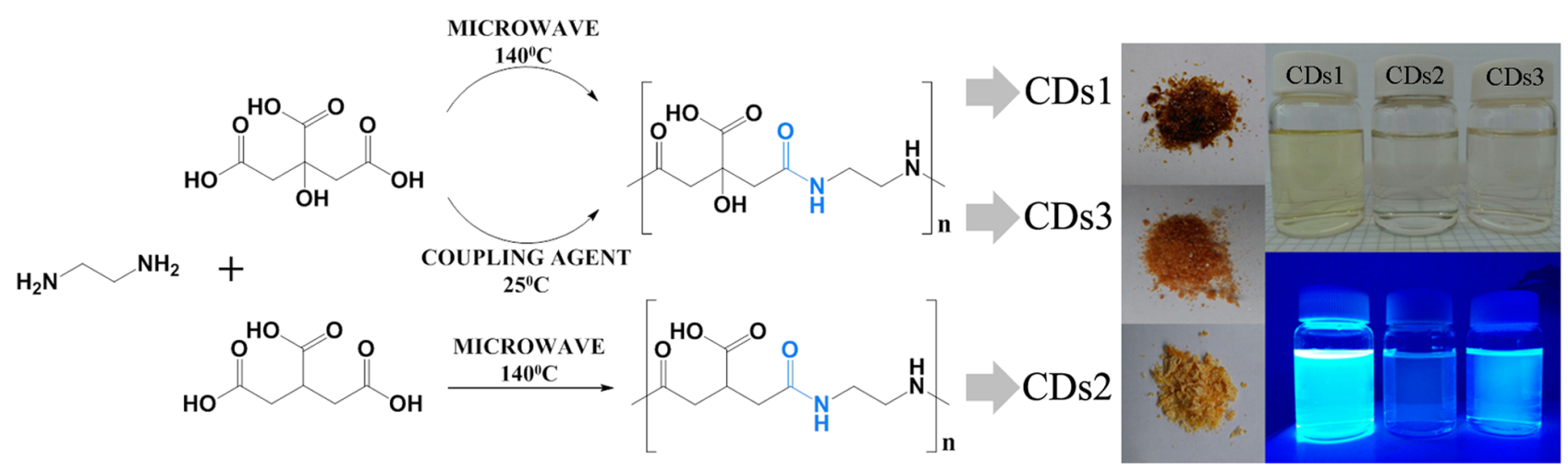

Figure 1. (Top) Reaction of EDA with CA through two synthetic pathways to form CDs1 and CDs3. (Bottom) Reaction of EDA with TA to form CDs2. (Right) Photographs of CDs1, CDs2 and CDs3 in solid and in water solution $(0.5 \mathrm{mg} / \mathrm{mL})$, with and without UV irradiation. 
resolved photoluminescence spectroscopy (Supporting Information, Figure S14). A peculiar absorption band in the range of $350-390 \mathrm{~nm}$ appears in the UV/Vis spectra of the three types of $\mathrm{CDs}$, which is directly related to the photoluminescence emission. In fact, the maximum emission intensity, found at $445 \mathrm{~nm}$ for CDs1 and CDs3 and at $470 \mathrm{~nm}$ for CDs2, is achieved by exciting exactly in the same region (360-390 nm depending on the CDs sample, see Figure 2b). Remarkably, emission spectra of CDs1, CDs2 and CDs3 taken at different excitation wavelengths do not reveal a wavelength dependency (Supporting Information, Figure S15). Although small shifts of the maximum position are found between the different types of CDs, their absorbance, excitation and emission are highly comparable and can be related to the same radiative process. The calculated photoluminescence lifetimes are also similar, being 14.7 ns for CDs1, 11.5 ns for CDs2 and $10.4 \mathrm{~ns}$ for CDs3. The fast lifetimes indicate that the radiative relaxation occurs by means of fluorescence. The quantum yield is very high for CDs1, reaching 64\%, and yet still remarkable in CDs2 (7\%) and in CDs3 (13\%) (Supporting Information, Figure S16). The higher quantum yield of CDs1 and CDs3 with respect to CDs2 should be related to the presence of the hydroxyl group. In fact, even if not directly involved in the fluorescence emission itself the ability of this group to form H-bonds may contribute to provide enhanced rigidity to the polymer structure and thus assists to suppress non-radiative decay channels.
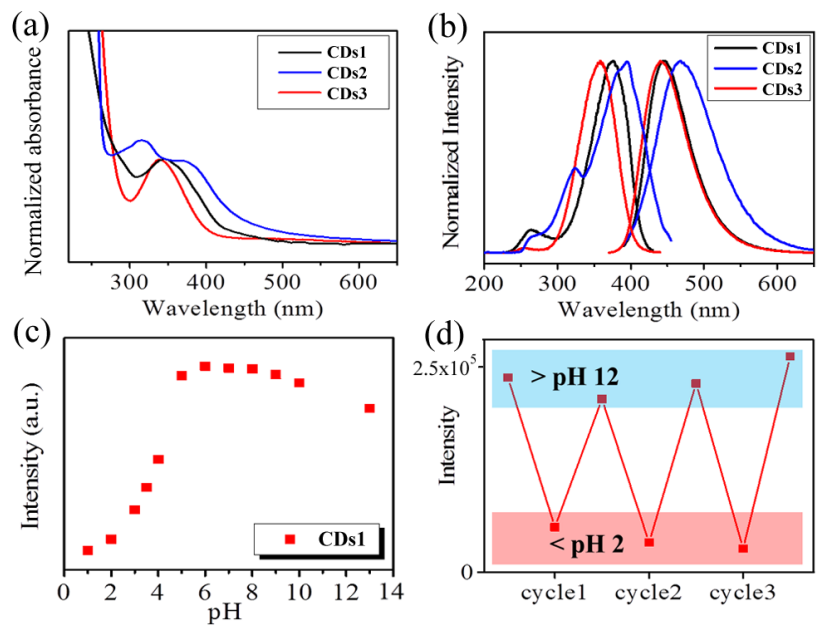

Figure 2. (a) UV/Vis, (b) Excitation and emission spectra of CDs1 (black), CDs2 (blue) and CDs3 (red). (c) pH dependence of the CDs1 fluorescence emission at $450 \mathrm{~nm}(\mathrm{ex}=370 \mathrm{~nm})$. (d) Fluorescence emission of CDs1 switching repeatedly the $\mathrm{pH}$ from basic to acidic conditions and vice-versa.

The optical properties of CDs1-CDs3 are very similar to those obtained from different amine and carboxylic acid

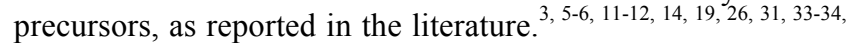
40-51 This coincidence implies a common principle behind the optical properties, suggesting the involvement of amides, amines and carboxylic acids. Consequently, any alteration of the polymer conformation thus should lead to a modification of the fluorescence behavior. Therefore, absorbance and emission measurements of CDs1 at various $\mathrm{pH}$ were performed. A highly basic solution of CDs1 ( $\mathrm{pH}>14)$ was treated dropwise with a $\mathrm{HCl}$ solution up to $\mathrm{pH}<1$. A sudden decrease in the absorbance and the emission of CDs1 is observed between pH 5 and pH 3 (Figure 2c, and Supporting
Information, Figure S17a, b). Switching the $\mathrm{pH}$ between basic and acidic conditions, a reversible loss of fluorescence intensity is detected, even during several cycles (Figure 2d, and Supporting Information, Figure S17c, d). These facts, ascribable to the carboxylic acid protonation-deprotonation, underline the role of $\mathrm{H}$-bonds and/or electrostatic forces in the interaction originating the fluorescence.

In order to shine light on the chemical interactions behind the fluorescence, a detailed structural characterization of CDs1, CDs2 and CDs3 was performed by means of elemental analysis, infrared spectroscopy, nuclear magnetic resonance spectroscopy and X-ray photoelectron spectroscopy.

Results of elemental analyses of CDs1 and CDs3 (Supporting Information, Figure S17 and Table S5) match with a formula of $\mathrm{C}_{8} \mathrm{H}_{12} \mathrm{~N}_{2} \mathrm{O}_{5}$, indicating that a stoichiometric copolymer is formed by condensation of CA and EDA in a 1:1 molar ratio. The same correspondence is found for CDs2 revealing a stoichiometric formula of $\mathrm{C}_{8} \mathrm{H}_{12} \mathrm{~N}_{2} \mathrm{O}_{4}$ (Supporting Information, Figure S18 and Table S5). These facts provide a good starting point for the understanding of the chemical structure of the formed CDs.

In the IR spectrum of CDs1 (Figure 3a) the typical $\mathrm{C}=\mathrm{O}$ stretching mode of the carboxylic group splits in two bands. The band at $1710 \mathrm{~cm}^{-1}$ reflects the carboxylic acid involved in $\mathrm{H}$-bond, while the band at $1780 \mathrm{~cm}^{-1}$ is the free form. The former exhibits a significantly higher intensity than the latter one. This indicates that almost all of the carboxylic groups are involved in H-bonds. The amide vibrational modes I and II are found at 1653 and $1560 \mathrm{~cm}^{-1}$, respectively. Their shifted position is comparable to the peptide bond vibrational modes and reflects the amide participation in H-bonds that increases the rigidity of the polymer. ${ }^{52-53}$ More features, such as $\mathrm{O}-\mathrm{H}$ and N-H stretching, are observed at $3500-2900 \mathrm{~cm}^{-1}$ and $\mathrm{C}-\mathrm{O}$ and C-N stretching at 1438, 1402, $1341 \mathrm{~cm}^{-1}$. Therefore, IR spectroscopy reveals that the structure of CDs1 encompasses a high involvement of $\mathrm{H}$-bonds and ionic supramolecular interactions that contribute to enhance the rigidity of the system. Identical considerations apply to the cases of CDs2 and CDs3, which both exhibit very similar IR profiles.

The XPS results of CDs1, CDs2 and CDs3 (Figure 3b) fit very well with the presence of the above-described structural features. The $\mathrm{C} 1 \mathrm{~s}$ peak was deconvoluted into five contributions (Figure 3c), the C-C (284.9 eV), C-N (285.7 $\mathrm{eV}), \mathrm{C}-\mathrm{OH}(286.3 \mathrm{eV}$, absent in CDs2), $\mathrm{N}-\mathrm{C}=\mathrm{O}(286.8 \mathrm{eV})$ and $-\mathrm{COOH}(288.2 \mathrm{eV})$. The $\mathrm{O} 1 \mathrm{~s}$ peak consists of three components corresponding to amide $(531.7 \mathrm{eV})$, carboxylic acid $(532.2 \mathrm{eV})$ and hydroxyl group (533.0 eV) (Supporting Information, Figure S19a-c). The N 1s peak is the sum of the amide $(399.4 \mathrm{eV})$ and the amine contribution (400.1 eV) (Supporting Information, Figure S19d-f). 
(a)

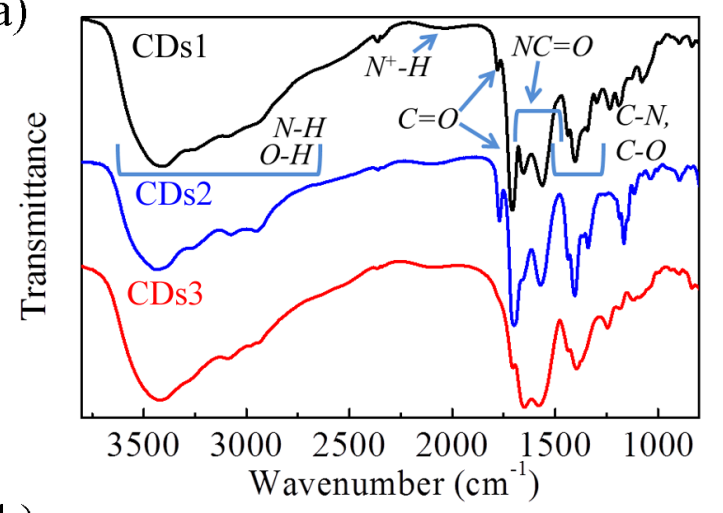

(b)

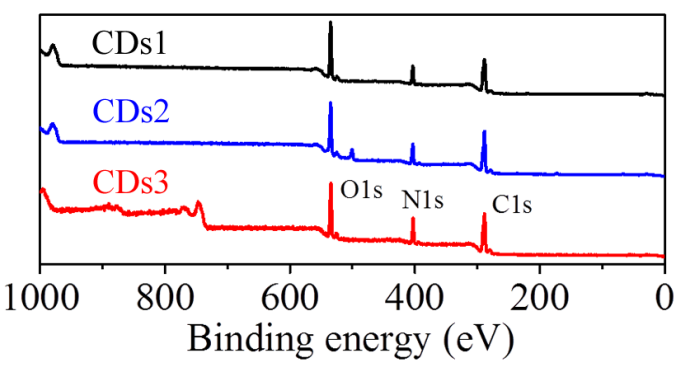

(c)
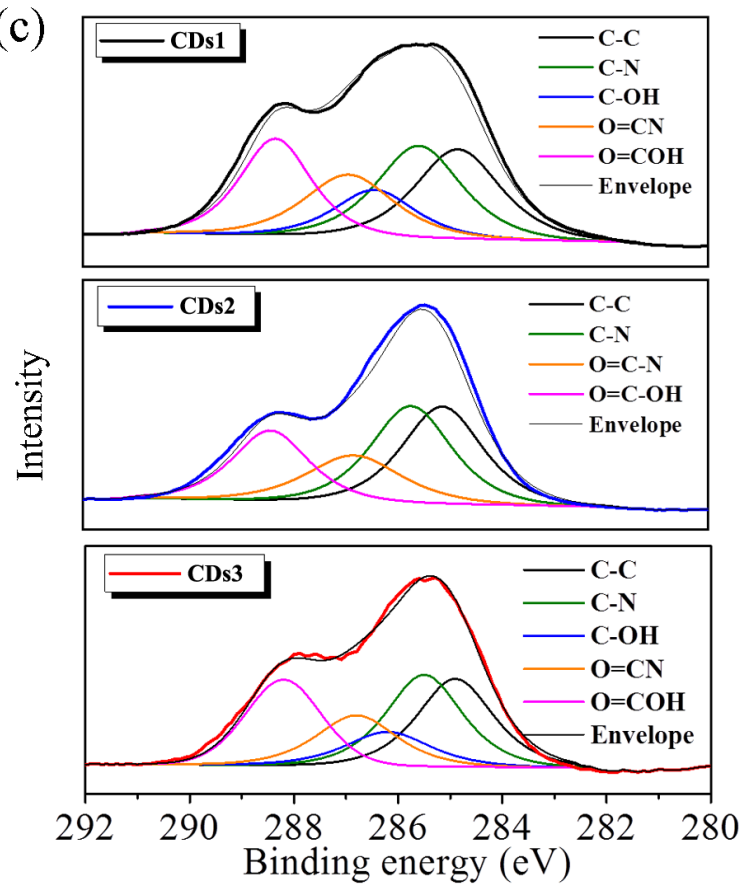

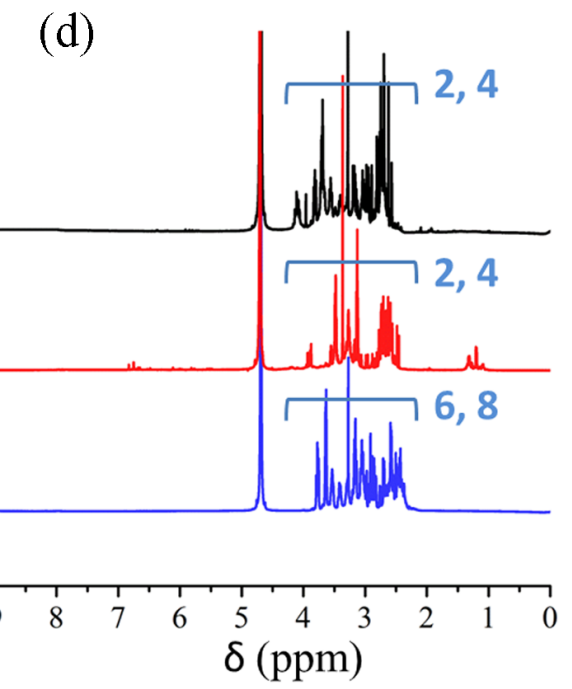

(e)

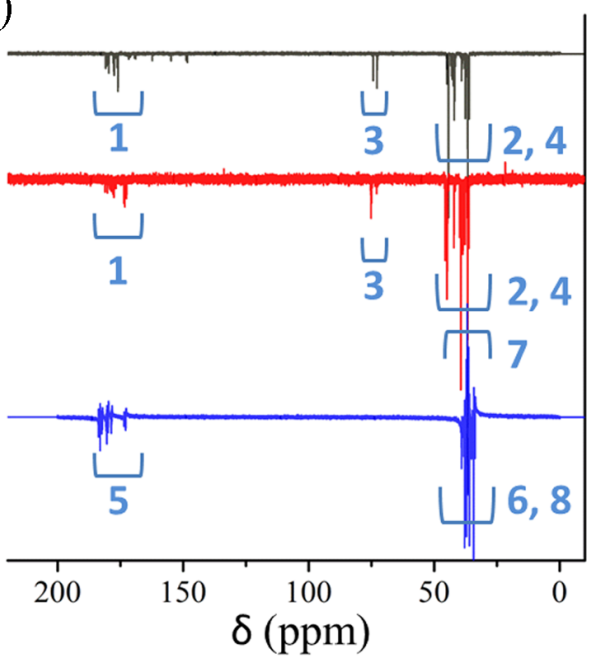

(f)

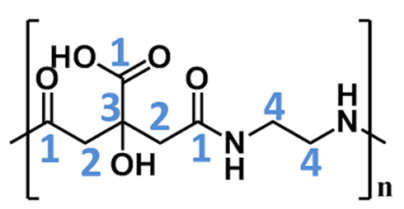<smiles>CCNCCNC(=O)CC(CC(C)C)C(=O)O</smiles>

Figure 3. (a) IR spectra and (b) XPS survey of CDs1 (black), CDs2 (blue) and CDs3 (red). (c) C1s spectra of CDs1 (top), CDs2 (center), CDs3 (bottom). (d) ${ }^{1} \mathrm{H}$ NMR and (e) ${ }^{13} \mathrm{C}\left\{{ }^{1} \mathrm{H}\right\}$ (APT) NMR of CDs1 (black), CDs2 (blue) and CDs3 (red). (f) Chain isomers for the signal attribution of (top) CDs1, CDs3, and (bottom) CDs2.

Proton $\left({ }^{1} \mathrm{H}\right)$ and carbon $\left({ }^{13} \mathrm{C}\right.$, APT: attached proton test $)$ standard NMR experiments, as well as two-dimensional correlation experiments ${ }^{1} \mathrm{H}_{-}{ }^{13} \mathrm{C}$ HSQC (heteronuclear singlequantum coherence) and ${ }^{1} \mathrm{H}_{-}{ }^{13} \mathrm{C}$ HMBC (heteronuclear multiple-bond correlation), of the CDs were measured in $\mathrm{D}_{2} \mathrm{O}$ and allowed to gather determinant information about the chemical structure and connectivity. In Figure $3 \mathrm{~d}$ and $3 \mathrm{e}$ a comparison of the ${ }^{1} \mathrm{H}$ and ${ }^{13} \mathrm{C}\left\{{ }^{1} \mathrm{H}\right\}$ APT NMR spectra of CDs1, CDs2 and CDs3 is shown. Despite the differences in the synthesis methods, the NMR profiles of CDs1 and CDs3 are remarkably similar, showing that in both cases the same type of polymer structure is obtained. The NMR spectra of CDs2 are also highly comparable, taking into account the differences due to the absence of the hydroxyl group. Merging the information obtained from all the NMR experiments, the chemical structure of CDs1, CDs2 and CDs3 is unambiguously identified as a non-conjugated polymer consisting of the product of condensation of CA and EDA for CDs1 and CDs3, and of TA and EDA for CDs2. The detailed analyses of all spectra for CDs1, CDs2 and CDs3, as well as the full assignment of all peaks, are provided in Supporting Information (Figures S20-22). The comparison of the NMR spectra also prove that unassigned minor features, specific to each of the employed synthetic route, are not common to all the samples, and thus cannot form the basis of the fluorescent behavior. Furthermore, NMR experiments provided valuable 
information regarding the conformation of the polymeric dots. In the ${ }^{1} \mathrm{H}$ NMR spectra, the sharp lineshape of the peaks (singlets and triplets) strongly suggests a compact and static structure, in fast motion with respect to the time of response of the technique. The ${ }^{1} \mathrm{H}-{ }^{13} \mathrm{C}$ HSQC spectra (Supporting Information, Figures S19c, S20c and S21c) show that the methylene carbons couple with a rather condensed set of proton signals. The high density of sharp signals is related to the variety of static chemical environments that surrounds these protons and can be explained with the presence of various chain isomers of the repetitive unit that coexist in the polymer, as well as with the existence of different ionized forms. These data highlight the branched and rigid conformation of the polymeric CDs.

In order to achieve a deeper understanding of the structure and photoluminescence of these nanoparticles, DFT calculations have been performed based on the CDs1 and CDs3 polymeric $\left[\mathrm{C}_{8} \mathrm{H}_{12} \mathrm{O}_{5} \mathrm{~N}_{2}\right]_{\mathrm{n}}$ units (see Supporting Information for details). For the analysis of the structural features that may be responsible of the CEE effect, clusters of different size have been studied, in particular the dimer $(n=$ $2)$, octamer $(n=8)$ and decamer $(n=10)$. The latter is a good approximation of the CDs1 real chain, whose molecular mass was measured to be $2300 \mathrm{Da}$ by size exclusion chromatography (SEC/RI, Supporting Information, Figure S4), while the polymeric unit weights $216 \mathrm{Da}$. The optimized structures show that the main characteristic is a very intricate network due to the establishment of both intra- and intermolecular hydrogen bonds. In Figure 4a two dimeric chains $(n=2)$ are represented and it is clear the degree of entanglement, both within and between chains. In Figure $4 \mathrm{~b}$ is represented one decameric chain $(n=10)$ that also shows a highly intricate structure due to the intramolecular hydrogen bonding (HB). This feature is reflected in the size of the nanoparticle. The calculated diameters are $1.564 \mathrm{~nm}$ and 1.670 $\mathrm{nm}$ for the octamer and the decamer, respectively, in good agreement with the particle size measured experimentally. (a)

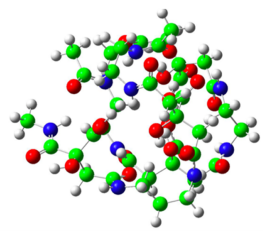

(c)

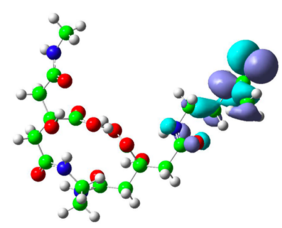

HOMO (b)

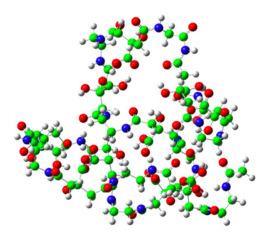

(d)

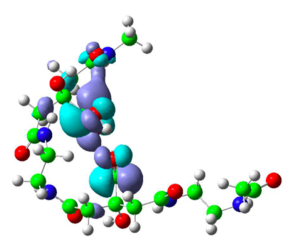

LUMO
Figure 4. Optimized molecular structures of (a) two dimer $(n=2)$ chains and (b) one decamer $(n=10)$ chain. (c) HOMO and (d) LUMO molecular orbitals involved in the fluorescence phenomenon.

As it has been previously mentioned, this aggregation of the chains is responsible of the enhancement of the fluorescence in these systems, due to a restriction of the vibrational or rotational degrees of freedom that may favor a non-radiative relaxation. In order to corroborate this statement, the optical properties of four representative systems have been calculated, namely, three dimers and one octamer. The first dimer has only one chain, while the others comprise two dimeric chains to generate two conformers, where either the intramolecular or the intermolecular HB is favored. The use of one or two chains, as well as two different conformers, allows studying the influence of the intra- and intermolecular interactions independently. The octamer has been used in order to relieve the computational effort. In Table 1 are gathered the absorption and emission energies, and the corresponding wavelengths.

Table 1. Absorption $\left(E_{a b s}\right)$ and emission $\left(E_{e m}\right)$ energies

\begin{tabular}{lll}
\hline & $E_{a b s}(\mathrm{eV})$ & $E_{e m}(\mathrm{eV})$ \\
\hline Dimer (one chain) & 5.1811 & --- \\
Dimer of two chains & 4.8794 & --- \\
(HB intramolecular) & & \\
$\begin{array}{l}\text { Dimer of two chains } \\
\text { (HB intermolecular) }\end{array}$ & 5.1382 & 1.8317 \\
Octamer & 4.6142 & 1.9602 \\
\hline
\end{tabular}

It is observed that the four models absorb in the UV, with wavelengths in the range of $\lambda_{\text {abs }}=239-269 \mathrm{~nm}$. The nature of the transition has been characterized by the analysis of the molecular orbitals involved, which is depicted in Figures 4c and $4 \mathrm{~d}$ for the dimer, in order to facilitate the visualization. The HOMO has its main contribution from the amide moiety (-CONH-), while the LUMO is centered in the carboxylic groups $(-\mathrm{COOH})$ and it is from HOMO to LUMO where excitation takes place. This means that HOMO and LUMO molecular orbitals are confined at very specific sites, that they are well separated, and that the fluorescence is a charge transfer process. This process is enhanced for the large models due to the sum of all local charge transfer processes occurring in each amide-carboxyl pair of the chain. Experimental evidence for these findings is provided by the results of our photoluminescence studies. First, the large Stokes-shift of about $1 \mathrm{eV}$ (see Figure 2b) should be related to a considerable change in the dipole moment between ground state and excited state, being consistent with the typical photo-induced charge transfer mechanism. ${ }^{54-57}$ Second, the protonation dependency of the emission intensity (see Figure $2 \mathrm{c}$ and S17) reveals the important role of the carboxylic acids in the fluorescence process: They affect the rigidity of the overall polymer network structure via hydrogen bonding or electrostatic interactions, as discussed above. Concomitantly, they act as electron acceptor in the charge transfer process. The observation of a strong decay of the fluorescence in an acidic environment is a consequence of the weakening of the carboxyl acids intramolecular interactions, which create the emitting state (see Figure 4c-d). On the contrary, strengthening the carboxylic intramolecular chain interactions, as obtained under neutral and basic conditions, leads to the recovery of the carboxylic acids intramolecular chain interactions, favoring charge-transfer and the fluorescence process.

Regarding the emission, a striking behavior is found. Both the dimer with one chain and that with two chains where the main interaction is the intramolecular HB, show transitions that are remarkably redshifted. In fact, the emission takes place in a region where the singlet excited state and the ground 
state potential energy surfaces cross, known as internal conversion (IC), involving a non-radiative relaxation and, hence, no fluorescence will be observed.

Considering the dimer with two chains, in which the intermolecular $\mathrm{HB}$ is favored, a clear redshift of the emission energy is observed with respect to the absorption, arising from a singlet excited state well separated from the ground state (1.8317 eV above the ground state). This emission takes place in the visible region $\left(\lambda_{\mathrm{em}}=676.9 \mathrm{~nm}\right)$. These results suggest that, in a small chain like a dimer, the crosslink generated by the intramolecular HB is not enough to avoid the non-radiative relaxation by vibration or rotation of the chain, and the main confinement is exerted by the intermolecular interactions. Nevertheless, this prominent feature disappears when longer chains are considered and a larger number of intramolecular interactions are allowed. Thus, inspecting the results for the octamer (single chain), a similar redshift is observed and the emission also take place in the visible $\left(\lambda_{\mathrm{em}}=632.5 \mathrm{~nm}\right)$. This means that the intramolecular crosslink is strong enough to hinder the vibration and rotation of the chain, and fluorescence is observed. At this point it is worthwhile to outline that the theoretical calculations are based on simplified models, which do not take into account several effects of experimental relevance, such as polymer branching, influence of additional chains, solvent effects, nor those related to the presence of free amines or charged states. These parameters contribute to enhance the rigidity of the polymer and thus may lead to further important down shifts of the calculated emission wavelengths towards the blue wavelength range. Importantly, despite quantitative shortfalls, our models yet provide a solid base to qualitatively explain the origin of the fluorescence of the CDs.

The identification of charge-transfer processes between welldefined and spatially separated functional groups as origin of the fluorescence in polymer CDs now provides a powerful toolbox towards an improved understanding of their use in technological applications. This is demonstrated at hand of studies on the sensing of metal ions in aqueous solutions. To this end, the fluorescence of an aqueous solution of CDs3 (0.4 $\mathrm{mg} / \mathrm{mL}$ ) was measured in the presence of several types of metal ions at a concentration of $1 \mathrm{mM}$. A high affinity (i.e. high quenching rate) towards $\mathrm{Fe}^{3+}, \mathrm{Cu}^{2+}$, and $\mathrm{Hg}^{2+}$, and other types of metal ions to a lower content is observed (Figure 5). Similar results were obtained for CDs1 and CDs2, which are also in agreement with those observed for CDs of unknown chemical structure. ${ }^{58-60}$ These findings clearly confirm that the high sensitivity can be ascribed to the common chemical structure of polymer CDs. In fact, the same groups identified to be involved in the charge transfer process can act also as chelating agents, in a similar way to EDA, EDTA and citrate. Carboxylic acids, amides and amines are therefore interacting strongly with the metal ions and the binding provides favorable non-radiative relaxation pathways, which compete with the intramolecular charge-transfer process and drastically reduce the photoluminescence emission. This study not only proves the existence of photoinduced charge-transfer processes between spatially separated amide and carboxylic moieties in polymer CDs, but also explains how to successfully exploit this mechanism in other types of applications of technological relevance.
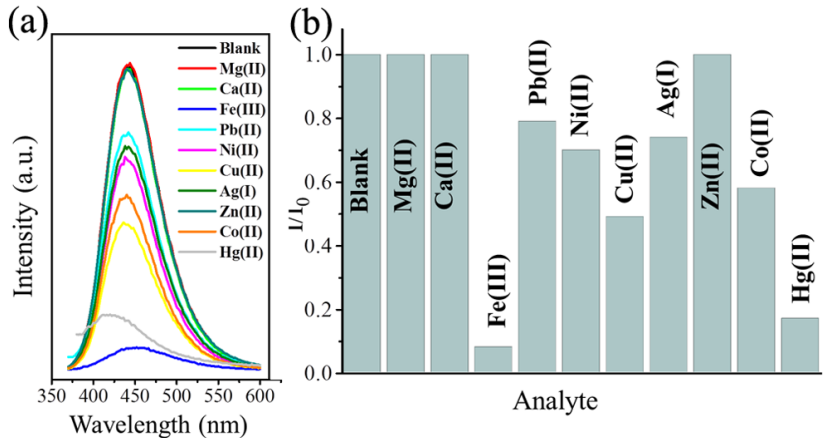

Figure 5. (a) Emission spectra of a CDs3 water solution in the presence of $1 \mathrm{mM}$ concentration of $\mathrm{Mg}^{2+}, \mathrm{Ca}^{2+}, \mathrm{Fe}^{3+}, \mathrm{Pb}^{2+}, \mathrm{Ni}^{2+}$, $\mathrm{Cu}^{2+}, \mathrm{Ag}^{2+}, \mathrm{Zn}^{2+}, \mathrm{Co}^{2+}, \mathrm{Hg}^{2+}$, (b) Relative fluorescence intensity of CDs 3 where I and Io are the intensities in presence and absence of the metal ions.

\section{CONCLUSIONS}

The synthesis of blue fluorescent model polymer CDs was obtained from CA and EDA as well as from TA and EDA by microwave irradiation, and from CA and EDA by a novel coupling agent-mediated condensation at room temperature. The optical features of the three types of model CDs of $1-1.5$ $\mathrm{nm}$ in size are remarkably similar, suggesting that the copolymerization of the reagents by amide bond is a sufficient condition for the formation of the fluorophore in polymer CDs. This hypothesis was further confirmed by a detailed structural characterization of the materials, which revealed that the presence of $\mathrm{H}$-bonds and electrostatic interactions goes along with the high conformational rigidity of the polymeric chain. DFT and TDDFT calculations of the octamer of this structure indeed show that supramolecular H-bond mediated interactions cause the rigid entanglement of the chains, hindering vibration and rotation, and facilitate the radiative relaxation process. Finally, the amide and the carboxylic groups are recognized as the moieties, which respectively provide the main contribution to the HOMO and the LUMO molecular orbitals. Photoinduced charge-transfer between these spatially separated groups, assisted by H-bond mediated supramolecular interactions in the entangled polyamide network therefore is identified as origin of the fluorescence phenomena in polymer carbon dots. We believe that this single emitter type process provides a universal explanation for the CDs' blue fluorescence observed in the vast and increasing amount of reports where these nanoparticles are prepared from carboxylic acids and amines. However, this process becomes masked by the contribution of additional fluorescent emitters, typically obtained when employing harsher conditions, resulting in an excitation dependent emission behavior. Our finding of a unifying principle so far well explains the performance of polymer CDs in existing technological applications. Moreover, it paves the way to exploit novel synthetic bottom-up routes to obtain polymer CDs with tailored properties uncovering yet unexploited opportunities.

\section{EXPERIMENTAL SECTION}

\section{Materials}

Citric acid anhydrous $(\geq 99.5 \%)$, tricarballylic acid (99\%), ethylendiamine $(99+\%)$, N,N'-diisopropylcarbodiimide $(99 \%)$, 
nickel (II) nitrate hexahydrate (98.5\%), iron (III) chloride $(97 \%)$, cobalt (II) nitrate hexahydrate $(98 \%)$, zinc (II) chloride $(\geq 97 \%)$, magnesium (II) nitrate hexahydrate $(99 \%)$, calcium (II) chloride dihydrate (99\%), copper (II) nitrate hemi(pentahydrate) (98\%), lead (II) nitrate ( $\geq 99 \%)$, mercurium (II) nitrate hydrate (98\%), were used without further purifications. Dialysis tubes with molecular weight cutoff (MWCO) 0.5-1 KDa were bought from Spectrum Labs.

Synthesis

Synthesis of CDs1. $2.0 \mathrm{~g}$ of citric acid monohydrate $(9.5$ mmol, 1 eq.) were dissolved in $15 \mathrm{~mL}$ of ultrapure water. Upon addition of $0.64 \mathrm{~mL}$ of EDA (1 equiv.) the solution was heated up to $140^{\circ} \mathrm{C}$ through microwave irradiation (stirring, open batch), provoking the evaporation of the water. The temperature was kept constant for one minute, after that the irradiation was stopped and the mixture redissolved in $10 \mathrm{~mL}$ of water. The same process was repeated two times more, for a total of three minutes at $140^{\circ} \mathrm{C}$. The solid product was diluted with ultrapure water, filtrated through a $0.45 \mu \mathrm{m}$ PTFE membrane and dialyzed against ultrapure water $(\mathrm{MWCO}=$ 0.5-1.0 KDa, 3 days, twice a day), yielding in a brownish powder, with a yeld in mass of $35 \%$.

Synthesis of CDs2. $2.0 \mathrm{~g}$ of tricarballylic acid (11.4 mmol, 1 equiv.) were dissolved in $15 \mathrm{~mL}$ of ultrapure water. Upon addition of $0.76 \mathrm{~mL}$ of EDA ( 1 equiv.) the solution was heated up to $140^{\circ} \mathrm{C}$ through microwave irradiation (stirring, open batch), provoking the evaporation of the water. The temperature was kept constant for 3 minutes, after that the irradiation was stopped and the mixture cooled down. The solid product was diluted with ultrapure water, filtrated through a $0.45 \mu \mathrm{m}$ PTFE membrane and dialyzed against ultrapure water $(\mathrm{MWCO}=0.5-1.0 \mathrm{KDa}, 3$ days, twice a day), yielding in a brownish powder with a yeld in mass of $27 \%$.

Synthesis of CDs3. $1.0 \mathrm{~g}$ of anhydrous citric acid (5.2 mmol, 1 equiv.) was dissolved in $5.0 \mathrm{~mL}$ of DMF. The solution was cooled in an ice bath and $2.6 \mathrm{~mL}$ of diisopropyl carbodiimide (DIC, 3 equiv.) were added. Subsequently, 0.35 $\mathrm{mL}$ of EDA (1 eq.) in $5 \mathrm{~mL}$ of water were slowly added and the reaction was stirred for 30 minutes at room temperature, during which the mixture turns its color from slightly yellow to red. The filtered reaction was stopped by adding slowly during a time of 3 hours a $\mathrm{NaOH}$ solution at $\mathrm{pH}=10$, until no formation of DIC-urea precipitate was observed. The crude was diluted in ultrapure water, filtered and washed with ethyl acetate. During these operations the coupling agent urea byproduct, in the form of a white precipitate, was removed. The water phase was dialyzed against ultrapure water (MWCO $=0.5-1.0 \mathrm{KDa}, 3$ days, twice a day). The dry product was obtained by freeze-drying, with a yield in mass of $29 \mathrm{wt}$ \%. It appears as a brownish powder.

Metal sensing. A $5 \mathrm{mM}$ aqueous solution of the metal was prepared. A water solution of $0.5 \mathrm{mg} / \mathrm{mL}$ of CDs3 was prepared. $1 \mathrm{~mL}$ of the metal solution was mixed with $4 \mathrm{~mL}$ of the CDs solution, and the fluorescence emission was measured. The blank was prepared adding $1 \mathrm{~mL}$ of water in the place of the metal solution.

\section{Instruments and characterization}

The microwave-assisted reaction was performed in a CEM Discover SP reactor employed in open-batch modality. Atomic force microscopy (AFM) images were acquired in air under ambient conditions using a NT-MDT Aura NTEGRA instrument operating in tapping mode at $110 \mathrm{kHz}$ resonance with $\mathrm{Au}$ tips HA NC ETALON (10nm curvature radius). Samples were prepared on silica substrates by drop casting of diluite water solutions. Particle height distribution analysis was carried out by using the Gwyddion software. Size exclusion chromatography was performed on a system composed by: pump (Izasa Scientific), automatic injector (Izasa Scientific), PL aquagel column OH-mixed-H (Agilent) and refractive index detector T-REX (Wyatt Technology). The mobile phase $(0.1 \mathrm{M}$ sodium acetate $)$ was flowed at $1 \mathrm{ml} / \mathrm{min}$ at $35^{\circ} \mathrm{C}$. For the calibration, PEO/PEG polymers were used in the range of $1970-44400 \mathrm{~g} / \mathrm{mol}$. The samples were dissolved in the mobile phase at concentration around $1 \mathrm{mg} / \mathrm{ml}$. The DLS measurements were recorded on a Malvern Nano Zetasizer $\mathrm{HT}$, on a $10 \mathrm{~mm}$ path-length plastic cuvette. Elemental analyses were performed in a Thermo Flash EA 1112 instrument with $\sim 3 \mathrm{mg}$ of powder samples. Infrared absorption measurements were performed on powder samples pressed with $\mathrm{KBr}$ into pellets with a Bruker Vertex 70 spectrometer. ${ }^{1} \mathrm{H}$ and ${ }^{13} \mathrm{C}$ NMR spectra were recorded in $\mathrm{D}_{2} \mathrm{O}$ solutions at $25{ }^{\circ} \mathrm{C}$ on a Bruker AV500 spectrometer ( $\delta$ in ppm and $\mathrm{J}$ in $\mathrm{Hz}$ ) at a ${ }^{1} \mathrm{H}$ NMR operating frequency of 500.13 $\mathrm{MHz} .{ }^{1} \mathrm{H}$ and ${ }^{13} \mathrm{C}$ NMR spectra were referenced using the solvent signal as an internal standard. The assignment of the ${ }^{1} \mathrm{H}$ NMR signals and the corresponding ${ }^{13} \mathrm{C}$ NMR peaks was carried out using standard ${ }^{1} \mathrm{H}-{ }^{13} \mathrm{C}$ edited-HSQC and ${ }^{1} \mathrm{H}-{ }^{13} \mathrm{C}$ $\operatorname{HMBC}\left(\mathrm{J}_{\mathrm{HC}}=8 \mathrm{~Hz}\right) 2 \mathrm{D}$-experiments. The determination of the diffusion coefficients $\mathrm{D}\left(\mathrm{m}^{2} / \mathrm{s}\right)$ was performed at controlled temperature $(300 \mathrm{~K})$ in spinning solutions of the corresponding compounds in $\mathrm{D}_{2} \mathrm{O}$ (concentrations about 2 $\mathrm{mM})$. The values of delta $(\delta)$ and delta $(\Delta)$ were optimized for each sample. In the case of $\delta$ the values found were in the range 1.7-2.0 $\mathrm{ms}$, while for $\Delta$ the optimized values ranged from $0.17-0.20$ s. X-ray photoelectron spectroscopy (XPS) measurements were taken with an ESCAPlus spectrometer using a $\mathrm{Mg}$ anode $(1253.5 \mathrm{eV})$ and a power of $225 \mathrm{~W}$. XPS data analysis was performed with casaXPS software. UV/Vis absorption spectra were recorded on a Shimadzu UV-2401 PC spectrophotometer. Photoluminescence excitation and emission spectra were recorded on a Horiba Jobin Yvon Fluoromax-P, slits of excitation and emission at $1 \mathrm{~mm}$. All the spectra were recorded at room temperature using $10 \mathrm{~mm}$ pathlength quartz cuvette. Pico-second time-resolved fluorescence spectra were measured by the time-correlated-single-photoncounting (TCSPC) method on a Nano-Log spectrofluorometer (Horiba JobinYvon), by using a laser diode as an excitation source (NanoLED, $375 \mathrm{~nm}$ ) and a UV-Vis detector TBX-PMT series $(250-850 \mathrm{~nm})$ by Horiba JobinYvon. Lifetimes were evaluated with the DAS6 Fluorescence-Decay Analysis Software.

\section{ASSOCIATED CONTENT}

Supporting information. Influence of the reaction time on the formation of CDs1: AFM, DLS, DOSY, SEC/RI, absorbance and fluorescence, fluorescence decay, elemental analysis, IR, XPS. Characterization of CDs1, CDs2, CDs3: AFM, DLS, DOSY, fluorescence decay, quantum yield, $\mathrm{pH}$ dependence, elemental analysis. XPS, NMR. Quantum chemical calculations.

\section{AUTHOR INFORMATION}

\section{Corresponding Author}

*Email: wmaser@icb.csic.es 


\section{Notes}

The authors declare no competing financial interest.

\section{ACKNOWLEDGMENTS}

This work has received funding from the European Union's Horizon 2020 research and innovation programme under the Marie Skłodowska-Curie grant agreement No 642742. AMB, and WKM further acknowledge Spanish MINEICO (project ENE2016-79282-C5-1-R), the Gobierno de Aragón (Grupo Reconocido DGA T03_17R), and associated EU Regional Development Funds). EPU acknowledges Gobierno de Aragón (Grupo Reconocido DGA E19_17R) and associated EU Regional Development Funds. Technical and human support provided by IZO-SGI, SGIker (UPV/EHU, MICINN, GV/EJ ERDF and ESF) is gratefully acknowledged for assistance and generous allocation of computational resources.

\section{ABBREVIATIONS}

APT, attached proton test; CA, citric acid; CDs, carbon dots; CEE, crosslink-enhanced emission; DFT, density functional theory; DLS, dynamic light scattering; DOSY, diffusion ordered spectroscopy; EDA, ethylenediamine; HB, hydrogen bond; $\mathrm{HMBC}$, heteronuclear multiple bond correlation; HOMO, highest occupied molecular orbital; HSQC, heteronuclear single quantum correlation; LUMO, lowest unoccupied molecular orbital; MWCO, molecular weight cut off; NMR, nuclear magnetic resonance; TA, tricarballylic acid; TDDFT, time dependent density functional theory; XPS, X-ray photoelectron spectroscopy.

\section{REFERENCES}

1. Liu, Q.; Zhang, N.; Shi, H.; Ji, W.; Guo, X.; Yuan, W.; Hu, Q. New J. Chem. 2018, 42 (4), 3097-3101.

2. Jiang, Y.; Han, Q.; Jin, C.; Zhang, J.; Wang, B. Mater. Lett. 2015, $141,366-368$.

3. He, J.; Zhang, H.; Zou, J.; Liu, Y.; Zhuang, J.; Xiao, Y.; Lei, B. Biosens. Bioelectron. 2016, 79, 531-535.

4. Kong, X.; Gong, Y.; Fan, Z. J. Fluoresc. 2016, 26 (5), 1755-1762.

5. Liu, Y.; Zhou, L.; Li, Y.; Deng, R.; Zhang, H. RSC Adv. 2016, 6 (110), 108203-108208.

6. Yang, M.; Li, B.; Zhong, K.; Lu, Y. J. Mater. Sci. 2018, 53 (4), 24242433.

7. Loo, A. H.; Sofer, Z.; Bouša, D.; Ulbrich, P.; Bonanni, A.; Pumera, M. ACS Appl. Mater. Interfaces 2016, 8 (3), 1951-1957.

8. Guo, X.; Xu, L.; Zhang, L.; Wang, H.; Wang, X.; Liu, X.; Yao, J.; Hao, A. J. Lumin. 2018, 196, 100-110.

9. Sarkar, T.; Bohidar, H. B.; Solanki, P. R. Int. J. Biol. Macromol. 2018, 109, 687-697.

10. Guo, R.; Chen, B.; Li, F.; Weng, S.; Zheng, Z.; Chen, M.; Wu, W.; Lin, X.; Yang, C. Actuator B-Chem 2018, 264, 193-201.

11. Wang, J.; Li, Q.; Zhou, J.; Wang, Y.; Yu, L.; Peng, H.; Zhu, J. Opt. Mater. 2017, 72, 15-19.

12. Xiao, Q.; Liang, Y.; Zhu, F.; Lu, S.; Huang, S. Microchim. Acta 2017, 184 (7), 2429-2438.

13. Sun, K.; Tang, Y.; Li, Q.; Yin, S.; Qin, W.; Yu, J.; Chiu, D. T.; Liu, Y.; Yuan, Z.; Zhang, X.; Wu, C. ACS Nano 2016, 10 (7), 6769-6781.

14. Kundu, A.; Lee, J.; Park, B.; Ray, C.; Sankar, K. V.; Kim, W. S.; Lee, S. H.; Cho, I.-J.; Jun, S. C. J. Colloid Interface Sci. 2018, 513, 505-514.

15. Khodadadei, F.; Safarian, S.; Ghanbari, N. Mater. Sci. Eng. C 2017, 79, 280-285

16. Sun, Y.; Cao, W.; Li, S.; Jin, S.; Hu, K.; Hu, L.; Huang, Y.; Gao, X.; Wu, Y.; Liang, X.-J. Sci. Rep. 2013, 3, 3036.

17. Zuo, G.; Xie, A.; Pan, X.; Su, T.; Li, J.; Dong, W. ACS Appl. Nano Mater. 2018, 1 (5), 2376-2385.

18. Min, Z.; Shi, L.; Jing, L.; Dan, Q.; Haifeng, Z.; Xingang, G.; Xiuli, H.; Zhigang, X.; Xiabin, J.; Zaicheng, S. Adv. Mater. 2014, 26 (21), 35543560 .

19. Zhang, F.; Feng, X.; Zhang, Y.; Yan, L.; Yang, Y.; Liu, X. Nanoscale 2016, $8(16), 8618-8632$.
20. Yuan, F.; Wang, Z.; Li, X.; Li, Y.; Tan, Z. a.; Fan, L.; Yang, S. Adv. Mater. 2017, 29 (3), 1604436.

21. Zhang, W.; Zhou, Y.; Dong, C.; Shen, B.; Xing, M.; Zhang, J. Res. Chem. Intermed. 2018, 1568-5675.

22. Han, M.; Zhu, S.; Lu, S.; Song, Y.; Feng, T.; Tao, S.; Liu, J.; Yang, B. Nano Today 2018, 19, 201-218.

23. Tao, S.; Zhu, S.; Feng, T.; Xia, C.; Song, Y.; Yang, B. Mater. Today Chem. 2017, 6, 13-25.

24. Zhu, S.; Song, Y.; Shao, J.; Zhao, X.; Yang, B. Angew. Chem. Int. Ed. 2015, 54 (49), 14626-14637.

25. Das, R.; Bandyopadhyay, R.; Pramanik, P. Mater. Today Chem. 2018, 8, 96-109.

26. Bagheri, Z.; Ehtesabi, H.; Rahmandoust, M.; Ahadian, M. M.; Hallaji, Z.; Eskandari, F.; Jokar, E. Sci. Rep. 2017, 7 (1), 11013.

27. Zhu, S.; Meng, Q.; Wang, L.; Zhang, J.; Song, Y.; Jin, H.; Zhang, K.; Sun, H.; Wang, H.; Yang, B. Angew. Chem. Int. Ed. 2013, 52 (14), 39533957.

28. Song, Y.; Zhu, S.; Zhang, S.; Fu, Y.; Wang, L.; Zhao, X.; Yang, B. J. Mater. Chem. C 2015, 3 (23), 5976-5984.

29. Zhu, S.; Zhao, X.; Song, Y.; Lu, S.; Yang, B. Nano Today 2016, 11 (2), 128-132.

30. Dhenadhayalan, N.; Lin, K.-C.; Suresh, R.; Ramamurthy, P. J. Phys. Chem. C 2016, 120 (2), 1252-1261.

31. Schneider, J.; Reckmeier, C. J.; Xiong, Y.; von Seckendorff, M.; Susha, A. S.; Kasák, P.; Rogach, A. L. J. Phys. Chem. C 2017, 121 (3), 2014-2022.

32. Shi, L.; Yang, J. H.; Zeng, H. B.; Chen, Y. M.; Yang, S. C.; Wu, C.; Zeng, H.; Yoshihito, O.; Zhang, Q. Nanoscale 2016, 8 (30), 14374-14378. 33. Fang, Q.; Dong, Y.; Chen, Y.; Lu, C.-H.; Chi, Y.; Yang, H.-H.; Yu, T. Carbon 2017, 118, 319-326.

34. Ehrat, F.; Bhattacharyya, S.; Schneider, J.; Löf, A.; Wyrwich, R.; Rogach, A. L.; Stolarczyk, J. K.; Urban, A. S.; Feldmann, J. Nano Lett. 2017, 17 (12), 7710-7716.

35. Zhu, S.; Song, Y.; Zhao, X.; Shao, J.; Zhang, J.; Yang, B. Nano Res. 2015, $8(2), 355-381$.

36. Zhao, E.; Lam, J. W. Y.; Meng, L.; Hong, Y.; Deng, H.; Bai, G.; Huang, X.; Hao, J.; Tang, B. Z. Macromolecules 2015, 48 (1), 64-71.

37. Qiao, Z. A.; Huo, Q.; Chi, M.; Veith, G. M.; Binder, A. J.; Dai, S. Adv. Mater. 2012, 24 (45), 6017-21.

38. Tao, S.; Song, Y.; Zhu, S.; Shao, J.; Yang, B. Polymer, 2018, 116, $472-478$

39. Tao, S.; Lu, S.; Geng, Y.; Zhu, S.; Redfern, S. A. T.; Song, Y.; Feng, T.; Xu, W.; Yang, B. Angew. Chem. Int. Ed. 2018, 57 (9), 2393-2398.

40. Gao, F.; Ma, S.; Li, J.; Dai, K.; Xiao, X.; Zhao, D.; Gong, W. Carbon 2017, 112, 131-141.

41. Zhang, Y.; Wang, Y.; Feng, X.; Zhang, F.; Yang, Y.; Liu, X. Appl. Surf. Sci. 2016, 387, 1236-1246.

42. Xu, Z.-Q.; Lan, J.-Y.; Jin, J.-C.; Dong, P.; Jiang, F.-L.; Liu, Y. ACS Appl. Mater. Interfaces 2015, 7 (51), 28346-28352.

43. Ogi, T.; Aishima, K.; Permatasari, F. A.; Iskandar, F.; Tanabe, E.; Okuyama, K. New J. Chem. 2016, 40 (6), 5555-5561.

44. Rong, M.; Feng, Y.; Wang, Y.; Chen, X. Actuators B-Chem. 2017, 245, 868-874.

45. Bhattacharyya, S.; Ehrat, F.; Urban, P.; Teves, R.; Wyrwich, R.; Döblinger, M.; Feldmann, J.; Urban, A. S.; Stolarczyk, J. K. Nat. Comm. 2017, $8(1), 1401$.

46. Tong, G.; Wang, J.; Wang, R.; Guo, X.; He, L.; Qiu, F.; Wang, G.; Zhu, B.; Zhu, X.; Liu, T. J. Mater. Chem. B 2015, 3 (4), 700-706.

47. Zhang, W.; Shi, L.; Liu, Y.; Meng, X.; Xu, H.; Xu, Y.; Liu, B.; Fang, X.; Li, H.-B.; Ding, T. RSC Adv. 2017, 7 (33), 20345-20353.

48. Hu, Y.; Yang, J.; Tian, J.; Yu, J.-S. J. Mater. Chem. B 2015, 3 (27), $5608-5614$

49. Wang, J.; Zhang, P.; Huang, C.; Liu, G.; Leung, K. C.-F.; Wáng, Y. X. J. Langmuir 2015, 31 (29), 8063-8073.

50. Sharma, A.; Gadly, T.; Neogy, S.; Ghosh, S. K.; Kumbhakar, M. Journal Phys. Chem. Lett. 2017, 8 (5), 1044-1052.

51. Zhang, Y.; Liu, X.; Fan, Y.; Guo, X.; Zhou, L.; Lv, Y.; Lin, J. Nanoscale 2016, 8 (33), 15281-15287.

52. Myshakina, N. S.; Ahmed, Z.; Asher, S. A. J. Phys. Chem. B 2008, $112(38), 11873-11877$.

53. Barth, A. Biochim. Biophys. Acta, Bioenerg. 2007, 1767 (9), 10731101.

54. Viallat, A.; Bom, R. P.; Cohen-Addad, J. P., Polymer 1994, 35 (13), 2730-2736. 
55. Wu, X.; Li, H.; Xu, Y.; Tong, H.; Wang, L., Polym. Chem. 2015, 6 (12), 2305-2311.

56. Mane, S. R.; Hsiao, I.-L.; Takamiya, M.; Le, D.; Straehle, U.; BarnerKowollik, C.; Weiss, C.; Delaittre, G., Small 2018, 14 (36), 1801571.

57. Wagner, K.; Crowe, L. L.; Wagner, P.; Gambhir, S.; Partridge, A. C.; Earles, J. C.; Clarke, T. M.; Gordon, K. C.; Officer, D. L., Macromolecules 2010, 43 (8), 3817-3827.

58. Zhou, M.; Zhou, Z.; Gong, A.; Zhang, Y.; Li, Q., Talanta 2015, 143, $107-113$.

59. Pengfei, L.; Yixin, Y.; Huimin, Z.; Jin, Z.; Zengyuan, P.; Kelong, A.; Yibing, C.; Qufu, W., Nanotechnology 2017, 28 (16), 165502.

60. Kong, X.; Gong, Y.; Fan, Z., J. Fluoresc. 2016, 26 (5), 1755-1762. 
Postprint: Unedited Author's version of Submitted Work published on-line on 13 Sept. 2018 in Journal of the American Chemical Society, DOI: 10.1021/jacs.8b06051

\section{Table of Contents}
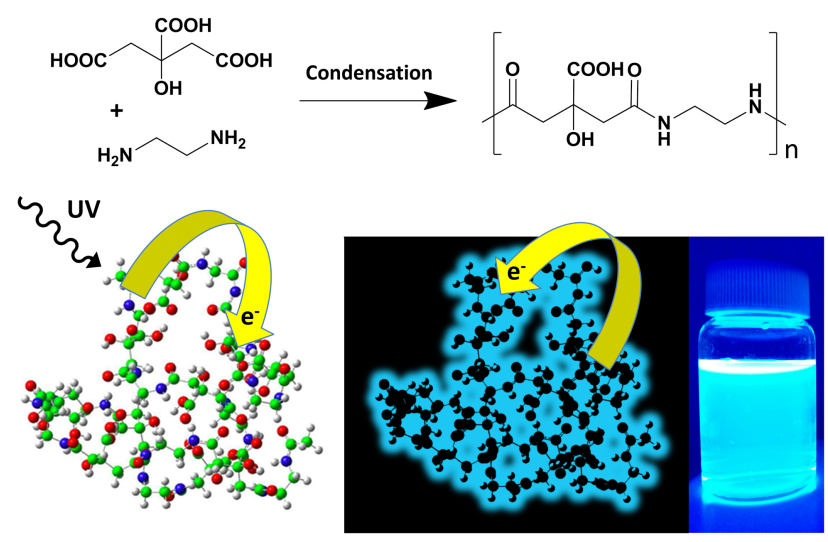